\title{
Honest, Open, Proud to support disclosure decisions and to decrease stigma's impact among people with mental illness: conceptual review and meta-analysis of program efficacy
}

\author{
Nicolas Rüsch ${ }^{1} \cdot$ Markus Kösters ${ }^{1}$
}

Received: 3 December 2020 / Accepted: 7 April 2021 / Published online: 24 April 2021

(c) The Author(s) 2021

\begin{abstract}
Purpose Honest, Open, Proud (HOP; formerly "Coming Out Proud"/COP) is a peer-led group program to support people with mental illness in their disclosure decisions and in their coping with stigma. The aims of this study were to provide (i) a conceptual review of HOP, including versions for different target groups and issues related to outcome measurement and implementation; and (ii) a meta-analysis of program efficacy.

Methods Conceptual and empirical literature on disclosure and the HOP program was reviewed. Controlled trials of HOP/ COP were searched in literature databases. A meta-analysis of HOP efficacy in terms of key outcomes was conducted.

Results HOP program adaptations for different target groups (e.g. parents of children with mental illness; veterans or active soldiers with mental illness) exist and await evaluation. Recruitment for trials and program implementation may be challenging. A meta-analysis of five HOP RCTs for adults or adolescents with mental illness or adult survivors of suicide attempts found significant positive effects on stigma stress $(\mathrm{smd}=-0.50)$ as well as smaller, statistically non-significant effects on self-stigma ( $\mathrm{smd}=-0.17)$ and depression $(\mathrm{smd}=-0.11)$ at the end of the HOP program. At 3- to 4-week follow-up, there was a modest, not statistically significant effect on stigma stress ( $\mathrm{smd}=-0.40,95 \%$-CI -0.83 to 0.04 ), while effects for selfstigma were small and significant $(\mathrm{smd}=-0.24)$. Long-term effects of the HOP program are unknown.

Conclusion There is initial evidence that HOP effectively supports people with mental illness in their disclosure decisions and in their coping with stigma. Implementation issues, future developments and public health implications are discussed.
\end{abstract}

Keywords Stigma $\cdot$ Self-stigma $\cdot$ Stigma stress $\cdot$ Disclosure $\cdot$ Honest, Open, Proud $\cdot$ Coming Out Proud $\cdot$ Peer intervention $\cdot$ Meta-analysis

\section{Introduction}

People with mental illness face the symptoms of their disorder as well as stigma and discrimination. The consequences for labeled individuals are significant and include status loss [1], disadvantages with respect to housing [2], work [3] or education, social isolation, poor health care and suicidality $[4,5]$. Many people with mental illness are not only aware of public prejudices, but turn them against themselves which is referred to as self-stigma ("I have a mental illness, therefore I must be lazy" [6]). Self-stigma is associated with a range

Nicolas Rüsch

nicolas.ruesch@uni-ulm.de

1 Department of Psychiatry II, Ulm University and BKH Günzburg, Ulm/Günzburg, Germany of negative outcomes [7] and leads to impaired self-esteem and a sense of demoralization, the so-called why try effect ("Why should I even try to achieve my goals? I am not worthy or not able" [8]). In longitudinal studies, self-stigma was a predictor of increased symptoms and of suicidality [7]. Stigma can also be a stressor if people with mental illness perceive stigma-related harm to be higher than their coping resources; this so-called stigma stress predicts self-stigma [5], poor quality of life, higher symptom levels and worse clinical outcomes $[9,10]$.

In this paper, which builds on previous work of Pat Corrigan and of our group [11,12], we will first outline existing approaches to reduce self-stigma, discuss the identity of being "mentally ill" and the role of disclosure decisions. We then present approach and content of the peer-led group program "Honest, Open, Proud" (HOP) for people with mental illness that focuses on disclosure decisions and offers a novel 
way to reduce self-stigma and stigma's impact, followed by an overview of current issues related to program delivery and a meta-analysis of HOP efficacy.

\section{Approaches to reduce self-stigma}

There are a range of programs to reduce self-stigma [13, 14]. In psychoeducational interventions, such as Ending SelfStigma developed by Patricia Lucksted and Amy Drapalski, participants learn facts that contradict their self-stigmatizing beliefs. However, there is limited evidence for the efficacy of this approach $[15,16]$. Cognitive therapies aim to correct self-stigma as a distorted self-concept, but their benefit is unclear [17]. Acceptance-based approaches use principles of acceptance and commitment therapy and mindfulness to reduce self-stigma and improve self-esteem [18]. Narrative programs help participants to develop more balanced stories of their lives; Narrative Enhancement and Cognitive Therapy, developed by Paul Lysaker, David Roe and Phil Yanos, is an example of a comprehensive narrative-cognitive strategy that reduces self-stigma [19]. Another approach to reduce self-stigma is to support people with mental illness in their disclosure decisions- this is the focus of the HOP program which will be discussed in more detail below. Mental illness being a discreditable stigma, as Erving Goffman put it [20], many face the decision whether and how to disclose their condition to others, and (non-) disclosure is a key reaction to stigma.

A recent meta-analysis examined the efficacy of interventions to reduce self-stigma across 14 controlled trials [13]; the majority of included studies investigated psychoeducational (five) or narrative (three) programs. On average, results were positive for psychoeducational and narrative interventions despite the small number of studies that were in part of poor quality. Findings were inconclusive for cognitive interventions. The conclusions of this meta-analysis were limited by the fact that it did not include all RCTs of interventions to reduce self-stigma published at that time [21].

Independent of the intervention strategy, self-stigma is nobody's fault nor is it a clinical problem, although clinicians should be aware of it as it can be a major barrier to service use, shared decision making and recovery [22-24]. Self-stigma is a consequence of public stigma and interventions should support people with mental illness to cope with self-stigma as long as public stigma exists.

\section{Identity of being "mentally ill"}

People with mental illness can react very differently to a psychiatric diagnosis. They may perceive it as a helpful concept or term that facilitates access to care and communication with clinicians. But diagnoses can also be seen as a stigmatizing label that attributes deficits to the person and reduces individuals to an illness without conveying a sense of hope [25]. There is the apparent paradox that insight, i.e. a greater awareness of having a mental illness, can be associated with better and with worse clinical or social outcomes. Paul Lysaker and colleagues found that if people with schizophrenia reject stigma as unfair, insight does not affect them negatively; however, if they suffer from self-stigma and agree with negative stereotypes about their group, greater insight leads to hopelessness [26]. Thus for an individual, to have an identity or self-concept as a person with mental illness is not bad or good per se-it depends on what the person associates with this identity.

\section{Disclosure}

People with mental illness often face the difficult choice whether to disclose their condition. Similar to the identity of having a mental illness, disclosure or non-disclosure is not good or bad per se- their consequences depend on the person and the environment [27]. In stigmatizing environments, the risks of disclosure often outweigh the benefits. A longitudinal study among long-term unemployed individuals that adjusted for clinical, work-related and socio-demographic variables provides an example: Those who were more willing to disclose their mental illness during their job search were less likely to be re-employed six months later [3]. However, greater comfort with disclosing to family and friends at baseline led to better quality of life over time [28]. All in all, the evidence suggests that disclosing one's mental illness improves well-being and social support [27]. However, disclosure decisions remain personal and complex because contexts and personal vulnerabilities differ: For some, not all, individuals secrecy leads to ruminations [27] and they may feel proud communicating their lived experience of mental illness and experiencing the authenticity that comes with disclosure [12]. Therefore, disclosure decisions are a key reaction to public and self-stigma and can affect selfstigma, stigma stress and well-being.

\section{Approach and aim of Honest, Open, Proud}

Considering the above-mentioned complexities of identity and disclosure and following previous work [29, 30], Corrigan and his colleagues in Chicago developed HOP as a peerled group program to help participants decide whether and how to disclose their mental illness. HOP's focus on disclosure decisions is based on models that highlight the role of (non-)disclosure for coping with the stigma associated with a concealable stigmatized identity $[31,32]$ and on findings that concealment versus disclosure is a dilemma for many people with mental illness [33]. These models highlight that disclosure decisions are a key component of successful 
coping with stigma and thus a mechanism to reduce stigma stress, self-stigma and their consequences, such as depression and reduced well-being or quality of life [34].

It is not HOP's goal to push people towards disclosure (although the program's English name has been misunderstood to suggest this). Non-disclosure in a stigmatizing environment can be a perfectly reasonable choice. HOP can lead to careful disclosure as well as to non-disclosure that is not driven by self-stigma or shame. HOP facilitator Chris White, Glasgow, called the latter outcome "empowered nondisclosure" (personal communication).

\section{HOP content}

HOP is a manualized program and consists of three lessons. Often HOP is therefore delivered in three two-hour sessions, with one session per week. Current HOP versions include a fourth booster session about a month after the third lesson.

In Lesson 1, participants first discuss how central mental illness is to their identity as well as possible reasons for disclosure (e.g. to find social support, to increase one's authenticity). The main part of Lesson 1 deals with benefits and risks of (non-)disclosure. Participants define their goals and what they expect after (non-)disclosure. At the end of the lesson, participants should be able to make a preliminary decision for or against disclosure in a certain setting.

Lesson 2 covers five levels of disclosure. Secrecy and disclosure are not all-or-nothing choices, but lie on a continuum between the extremes of social withdrawal (to avoid that others find out about one's mental illness) and active broadcasting of one's lived experience. In between are secrecy or non-disclosure (without social withdrawal), selective disclosure (of certain information to selected individuals), and indiscriminate disclosure (without active broadcasting). Lesson 2 also helps to prepare disclosure decisions. Participants learn how to "test the waters" with potential addressees of their disclosure. A participant could, outside the HOP group session, talk to a colleague about a TV series she saw last night that portrayed a person with mental illness. If the colleague responds with stigmatizing comments, she will be warned to be careful with disclosing to this colleague.

Lesson 3 develops ways to tell one's story. The workbook offers an outline to develop one's story that includes experiences with the (beginning) mental illness, dark days, recovery and experiences how to overcome stigma and discrimination. Lesson 3 ends by reflecting on what participants have learnt in the program and how peer support can help them to move on. In Lesson 4, as a booster session, participants recall their attitudes towards disclosure at the end of Lesson 3 and discuss their experiences with (non-)disclosure in the meantime, incl. whether that has changed their choices regarding disclosure or the stories they would like to tell.

\section{Aims of this study}

There are a number of recent systematic reviews on interventions to reduce self-stigma among people with mental illness [13, 14, 35-37]. Support with disclosure decisions is a novel strategy to reduce self-stigma. The approach has been outlined in conceptual work on HOP [12], and shorter pieces have commented on different aspects of HOP [38, 39]. However, we are not aware of a review or meta-analysis that specifically deals with HOP's efficacy, and the most recent review of programs to reduce self-stigma [13] was incomplete with regard to published HOP trials [21]. A synthesis of available evidence is therefore needed to guide future work on HOP's evaluation and implementation.

This paper had two aims, (i) to provide a conceptual narrative review on the HOP program incl. issues related to different versions and program implementation; and (ii) to provide a meta-analysis on the efficacy of the HOP program based on controlled trials. Both aims are interconnected: In case the meta-analysis provides evidence that HOP is beneficial, issues of program delivery in different settings become more important (Table 1).

\section{Methods}

First, we non-systematically surveyed conceptual and empirical literature to inform our narrative review on disclosure, mental illness and the HOP intervention, incl. HOP training materials and trial registries for ongoing HOP trials. Second, we searched for controlled trials of the HOP program (previously known as COP/Coming Out Proud) program, randomized or not randomized, in Pubmed, Medline and PsycInfo on November 15, 2020, and again on February 9, 2021, for our meta-analysis. "Honest, Open, Proud", "Coming Out Proud", and "In Würde zu sich stehen" (IWS, the program's German name) were used as search terms. English and German language articles were searched without time limit. Reference lists of trial publications were checked, and colleagues active in HOP research were contacted. All controlled trials of COP or HOP were included in the metaanalysis while uncontrolled pre-post evaluations were not. The search was run by NR and checked by MK; data were extracted from RCT datasets by NR and discussed with MK. We extracted data on the above-mentioned outcomes (Table 2) as well as on study and sample characteristics (Table 3) and, if available, on feasibility and implementation. 
Table 1 Versions of the HOP program for specific target groups; versions with different names than HOP add “...—a HOP program" to their name (e.g. "2Share-a HOP program" to clarify that 2Share is a HOP version)

\begin{tabular}{|c|c|c|c|}
\hline Target group & Program name & Lead program developers & Evaluations \\
\hline Adults with mental illness & $\begin{array}{l}H O P \text { (the original standard version } \\
\text { from which other versions are } \\
\text { derived) }\end{array}$ & Patrick W. Corrigan, Chicago, US & $\begin{array}{l}2 \text { RCTs }[47,48], 1 \\
\text { feasibility study [51] }\end{array}$ \\
\hline Adolescents with mental illness & $H O P$ or $U p$ to $M e$ & $\begin{array}{l}\text { Sarah Reed, Sue McKenzie \& Suzette } \\
\text { Urbashich, Wisconsin, and Pat Cor- } \\
\text { rigan, Chicago, US }\end{array}$ & $1 \mathrm{RCT}[21]$ \\
\hline College students with mental illness & HOP-College & $\begin{array}{l}\text { Maya Al-khouja and P.W. Corrigan, } \\
\text { Chicago, US }\end{array}$ & 1 RCT [49] \\
\hline $\begin{array}{l}\text { Parents of children with mental health } \\
\text { challenges }\end{array}$ & Starting the Conversation & Jeneva Ohan, Perth, Australia & - \\
\hline Suicide attempt survivors & 2Share & Lindsay Sheehan, Chicago, US & $1 \mathrm{RCT}[46]$ \\
\hline Soldiers with mental illness & HOP & Nicolas Rüsch, Ulm, Germany & Pilot RCT ongoing ${ }^{\mathrm{a}}$ \\
\hline Veterans with mental illness & $H O P$ & $\begin{array}{l}\text { Michelle Andra, Veterans' Health } \\
\text { Administration, and Jon Larson, } \\
\text { Chicago, US }\end{array}$ & - \\
\hline $\begin{array}{l}\text { Mental health professionals with } \\
\text { mental illness }\end{array}$ & $\begin{array}{l}H O P-M H P \\
\text { (delivered as anonymous online self- } \\
\text { help guide) }\end{array}$ & Katrina Scior, London, UK & Pilot RCT completed ${ }^{\mathrm{b}}$ \\
\hline People with Tourette Syndrome & $\begin{array}{l}\text { HOP: To Eliminate the Stigma of } \\
\text { Tourette }\end{array}$ & $\begin{array}{l}\text { Sarah Reed, Sue McKenzie \& Ellie } \\
\text { Jarvie, Wisconsin, US }\end{array}$ & - \\
\hline People with substance use disorders & $H O P-S U D$ & Patrick W. Corrigan, Chicago, US & - \\
\hline People with urinary incontinence & To Tell or Not to Tell & Lindsay Sheehan, Chicago, US & - \\
\hline $\begin{array}{l}\text { People with dementia and their signifi- } \\
\text { cant others }\end{array}$ & Who to Tell, How and When? & Jemini Bhatt, London, UK & 1 feasibility study $[50]$ \\
\hline People with psychosis & $\begin{array}{l}\text { Let's talk } \\
\text { (delivered as 1-to-1 peer support) }\end{array}$ & Melissa Pyle, Manchester, UK & Pilot RCT ongoing ${ }^{\mathrm{c}}$ \\
\hline
\end{tabular}

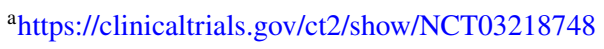

${ }^{b}$ http://www.isrctn.com/ISRCTN18418155

${ }^{\mathrm{c}} \mathrm{https}$ //www.fundingawards.nihr.ac.uk/award/NIHR200460

\section{Meta-analysis}

We selected three outcome domains for the meta-analysis: stigma stress, self-stigma, and depressive symptoms. For each outcome domain, we planned to choose one measure, if possible, that was used in all trials. Effect sizes were calculated as standardized mean differences (Hedges' g), comparing the means of the HOP intervention group to the treatment as usual group at post intervention and follow-up assessments. For the purpose of this meta-analysis, principal investigators of HOP trials gave us access to the individual participant data of all included trials. For data that had not been published or for outcomes that had been reported with different scorings (e.g. sum scores instead of mean scores), we computed the mean scores for stigma stress, depression and self-stigma from the RCT data sets such that each outcome was on the same scale and could be compared across trials.

Sometimes change scores from baseline rather than postbaseline scores are used to account for baseline differences between intervention and control groups or for problems with randomization. However, this approach is not recommended [40] and less conservative [41], therefore our metaanalysis is based on post-baseline scores. Effect sizes are considered small in the range of 0.2 , medium at about 0.5 and large at values of 0.8 . Due to differences between samples or study designs, statistical heterogeneity of effect sizes had to be expected. Therefore, effect sizes were combined within a random effects model. Heterogeneity of effect sizes was assessed with the $I^{2}$ parameter. $I^{2}$ is a descriptive statistic of the percentage of the variability in effect estimates that is due to heterogeneity rather than chance; the higher the $I^{2}$ values from 0 to $100 \%$, the higher the heterogeneity. All analyses were conducted with RevMan 5 [42].

\section{Results}

\section{HOP versions for different target groups}

The original HOP version for adults with mental illness has been adapted for different cultural contexts and target groups 


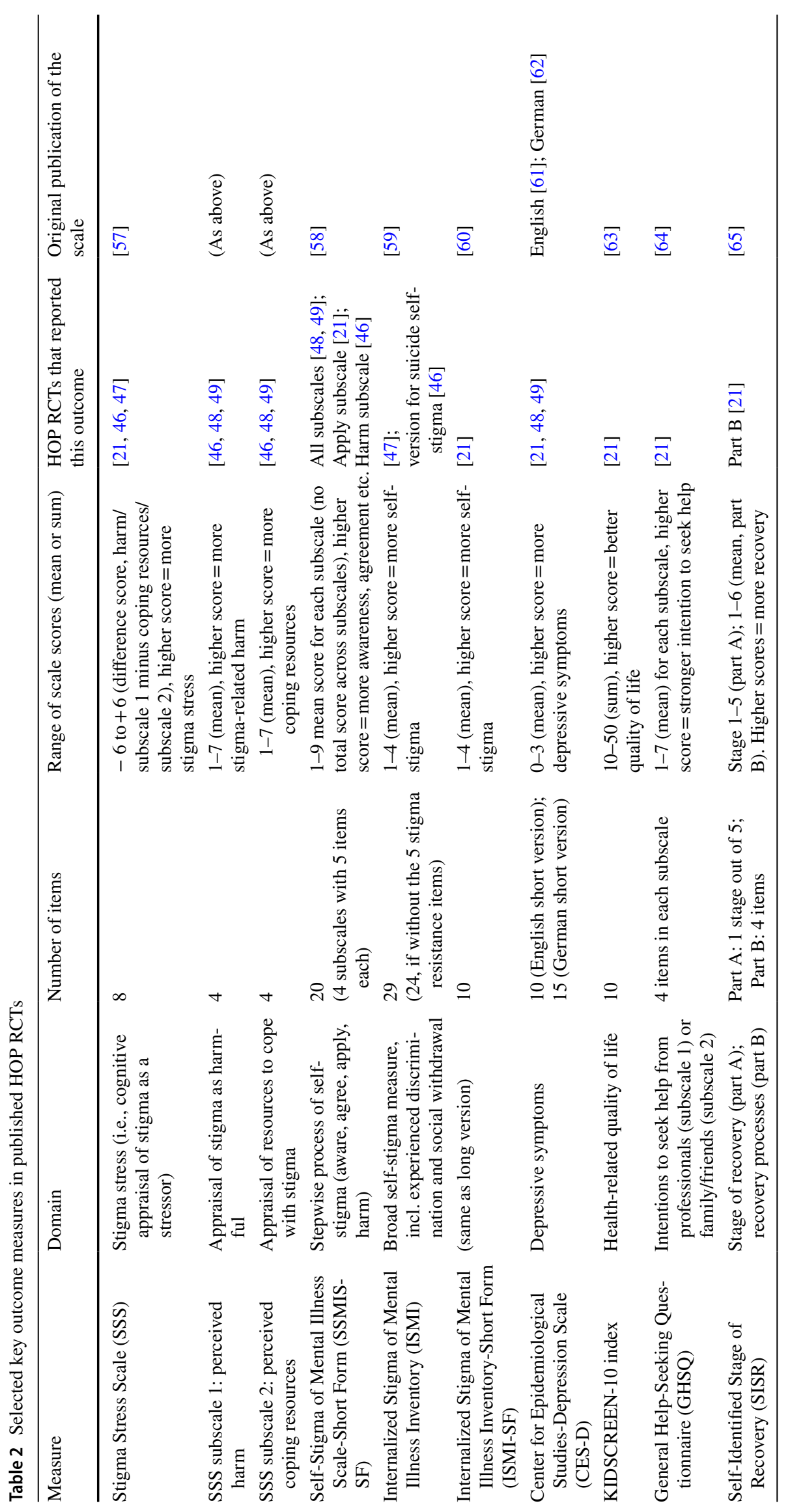




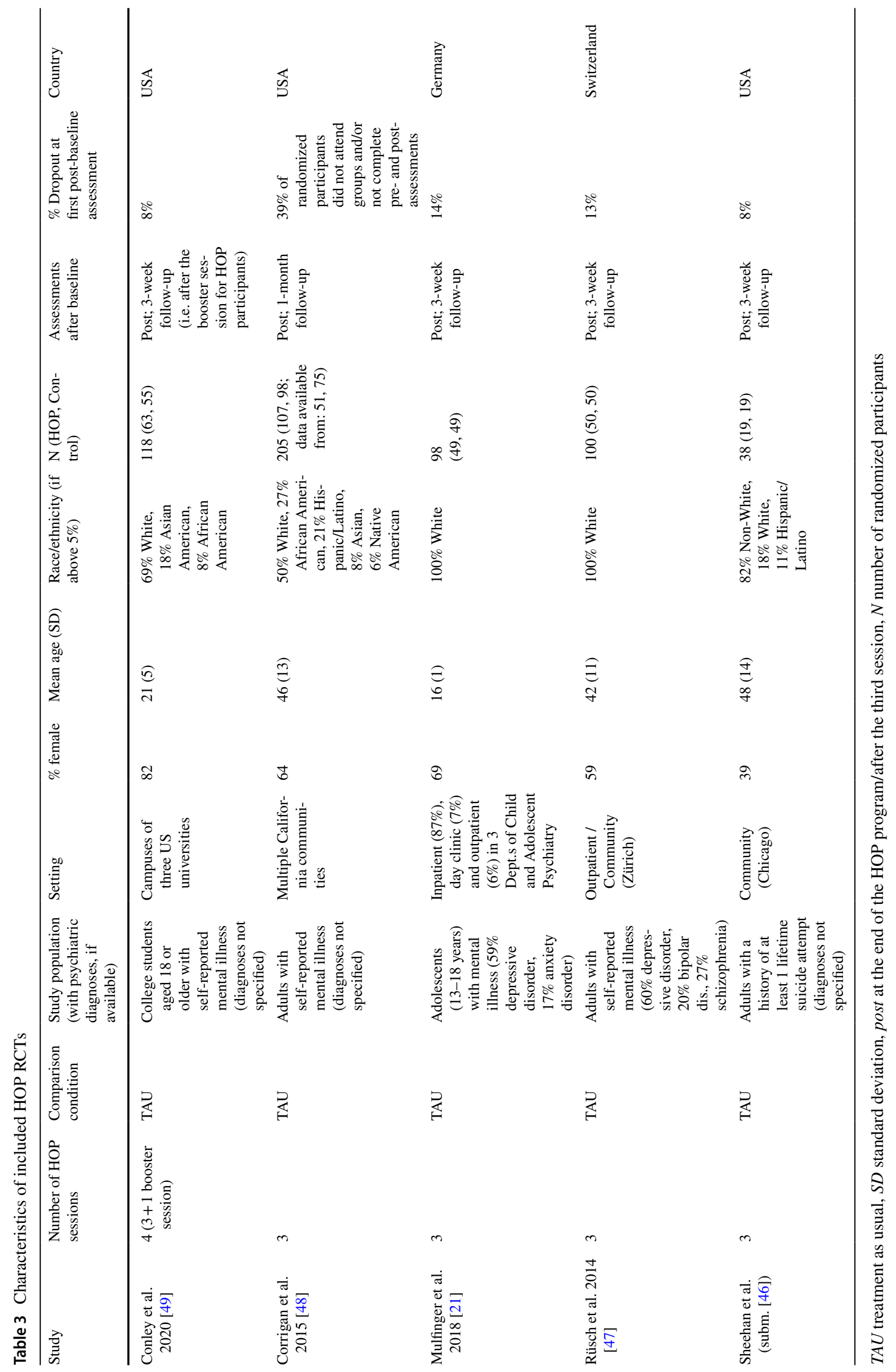


(Table 1). In the spirit of participatory research, HOP was adapted in cooperation between the target group and other relevant stakeholders because stigma varies between cultures and different conditions [39]. For example, to create a German HOP version for adolescents with mental illness, the adolescent version developed in the US was first translated. Based on focus groups and discussions with adolescents with and without mental illness, teachers, parents and mental healthcare providers, the content was then culturally adapted [21, 43, 44]. Information about HOP versions is available in English (hopprogram.org) and German (uni-ulm.de/med/ iws/) and from lead developers listed in Table 1.

\section{Group facilitators, participants, program fidelity}

\section{HOP group facilitators}

HOP groups are facilitated by peers that is by people with lived experience of mental illness. This lived experience makes group facilitators credible for participants. It is also helpful for group facilitators to share a specific background that affects disclosure decisions. For example, in a current trial of HOP for active soldiers in Germany, groups are facilitated by soldiers with mental illness who understand the specific pros and cons of disclosing in the military. Regarding the choice of group facilitators, there is a degree of flexibility. In our trial of HOP for adolescents in inpatient settings, groups were run by a young adult peer and a young mental health professional [21]. Regarding the number of group facilitators, two peers often run a group together. Group facilitators are usually trained in a two-day workshop, followed by supervision as needed.

\section{HOP participants}

It is not necessary that participants have received a psychiatric diagnosis or treatment although this is usually the case. They should have personal experience with mental health problems and have dealt with disclosure decisions. Groups ideally consist of four to eight participants. If the number is higher or smaller, a lively exchange between group members can be difficult. Finally, participants should not join the group after the first session.

\section{Fidelity}

In the RCTs, program fidelity was assessed by a checklist. Research assistants sat in the background of each session and noted whether key content, e.g. a worksheet with group discussion, had been covered in each lesson. Fidelity was high in the range of 80-90\%. Outside the research context, it is a challenge to make the program available and boost its implementation while maintaining satisfactory quality of program delivery. A current Australian study addresses this question with an implementation science framework and collects qualitative and quantitative data to assess the feasibility of implementing HOP [45]. With respect to preparing peers for running groups, HOP follows a train-the-trainer approach: Interested individuals are trained in centers with HOP expertise. Trained group facilitators can then run HOP groups and learn from the exchange with other group facilitators as well as from HOP master trainers as supervisors.

\section{HOP trials}

Our literature search identified no non-randomized controlled trials and four randomized controlled trials of HOP (or COP, its previous name); data from a fifth RCT, submitted for publication, was kindly provided by Lindsay Sheehan, Chicago [46]. All trials had a similar study design (see Table 3 for characteristics of included RCTs). The first trial in Switzerland found short-term benefits on stigma stress, secrecy and disclosure-related distress that were only partly sustained at three-week follow-up [47]. A Californian RCT reported benefits on stigma stress appraisals, self-stigma and, among women, on depression that were mostly sustained at one-month follow-up [48].

An RCT of HOP for adolescents in Southern Germany showed positive effects post intervention and at follow-up on stigma stress, self-stigma, quality of life, depression, recovery and help-seeking attitudes [21]. The effect on quality of life at follow-up was mediated by reduced stigma stress after the intervention. In a preliminary economic analysis, HOP program delivery costs were much lower than average mental health service costs, and HOP was cost-efficient in terms of quality of life gains (appr. €7.000-€20.000 per QALY, depending on whether costs of HOP facilitator training were included [21]). Fourth, an RCT among US college students showed benefits on self-stigma, self-efficacy in terms of disclosing and on resources to cope with stigma; this study was the only one to offer a fourth booster session [49]. The fifth and yet unpublished RCT evaluated the 2Share HOP version among suicide attempt survivors in Chicago and found positive effects on self-stigma and depression after the intervention [46].

We identified one HOP RCT among adults with mental illness, led by Winnie Mak in Hong Kong; it was unpublished and data were not yet available for the meta-analysis (www.2.ccrb.cuhk.edu.hk/registry/public/615/history/2452). Katrina Scior and her colleagues in London, UK, recently completed a pilot study of their HOP version for mental health professionals, but results are yet unpublished (ucl. ac.uk/pals/hop-mhp-project). Jem Bhatt and colleagues in London, UK, found in a feasibility study that their HOP version for people living with dementia appeared helpful based on qualitative participant feedback, but recruitment was 


\begin{tabular}{|c|c|c|c|c|c|c|c|c|}
\hline \multirow[b]{2}{*}{ Study or Subgroup } & \multicolumn{3}{|c|}{ HOP } & \multicolumn{3}{|c|}{ TAU } & \multicolumn{2}{|c|}{ Std. Mean Difference } \\
\hline & Mean & $S D$ & Total & Mean & $S D$ & Total & Weight & IV, Random, $95 \% \mathrm{C}$ \\
\hline \multicolumn{9}{|l|}{ Stigma stress } \\
\hline Conley 2020 & -1.52 & 1.79 & 53 & -0.55 & 1.98 & 54 & $22.2 \%$ & $-0.51[-0.90,-0$. \\
\hline Corrigan 2015 & -1.16 & 2.09 & 45 & -1.26 & 2.51 & 67 & $22.4 \%$ & $0.04[-0.34,0$ \\
\hline Mulfinger 2018 & -2.33 & 1.91 & 39 & -0.29 & 2.01 & 45 & $20.1 \%$ & $-1.03[-1.49,-0$. \\
\hline Rüsch 2014 & -1.27 & 2.29 & 39 & -0.23 & 1.85 & 47 & $20.9 \%$ & $-0.50[-0.93$, \\
\hline Sheehan (submitted) & -3.39 & 2.55 & 16 & -1.87 & 2.33 & 19 & $14.5 \%$ & $-0.61[-1.2$ \\
\hline Subtotal $(95 \% \mathrm{Cl})$ & & & 192 & & & 232 & $100.0 \%$ & $-0.50[-0.87$, \\
\hline \multicolumn{9}{|c|}{ Heterogeneity: Tau $^{2}=0.12 ; \mathrm{Chi}^{2}=13.02, \mathrm{df}=4(P=0.01) ; 1^{2}=69 \%$} \\
\hline \multicolumn{9}{|l|}{ Perceived resources } \\
\hline Conley 2020 & 5.42 & 1.05 & 53 & 4.79 & 1.27 & 54 & $23.3 \%$ & $0.54[0.15,0$. \\
\hline Corrigan 2015 & 5.54 & 1.08 & 45 & 5.37 & 1.25 & 67 & $23.7 \%$ & $0.14[-0.24,0$. \\
\hline Mulfinger 2018 & 5.12 & 1.12 & 39 & 4.04 & 1.07 & 45 & $19.9 \%$ & $0.98[0.52,1$. \\
\hline Rüsch 2014 & 5.07 & 1.16 & 39 & 4.4 & 1.27 & 47 & $21.0 \%$ & $0.54[0.11,0$ \\
\hline Sheehan (submitted) & 6.17 & 1.01 & 16 & 5.47 & 1.06 & 19 & $12.0 \%$ & $0.66[-0.03$, \\
\hline Subtotal $(95 \% \mathrm{Cl})$ & & & 192 & & & 232 & $100.0 \%$ & $0.55[0.26,0$ \\
\hline
\end{tabular}

Heterogeneity. Tau ${ }^{2}=0.05 ; \mathrm{Ch}^{2}=7.91, \mathrm{df}=4(\mathrm{P}=0.09) ; 1^{2}=49 \%$

Test for overall effect: $Z=3.80(P=0.0001)$

\section{Perceived stigma-related harm}

$\begin{array}{lrrrrrrr}\text { Conley 2020 } & 3.9 & 1.5 & 53 & 4.24 & 1.5 & 54 & 23.4 \% \\ \text { Corrigan 2015 } & 4.38 & 1.97 & 45 & 4.11 & 1.87 & 67 & 23.5 \% \\ \text { Mulfinger 2018 } & 2.79 & 1.33 & 39 & 3.75 & 1.55 & 45 & 20.3 \% \\ \text { Rüsch 2014 } & 3.8 & 1.58 & 39 & 4.17 & 1.44 & 47 & 21.0 \% \\ \text { Sheehan (submitted) } & 2.78 & 1.89 & 16 & 3.61 & 1.74 & 19 & 11.9 \% \\ \text { Subtotal (95\% Cl) } & & & 192 & & & 232 & 100.0 \%\end{array}$

Heterogeneity. Tau $^{2}=0.05 ; \mathrm{Chi}^{2}=7.66, \mathrm{df}=4(\mathrm{P}=0.10) ; \mathrm{I}^{2}=48 \%$

Test for overall effect: $Z=1.84(P=0.07)$

\section{Self-stigma}

Conley 2020

Corrigan 2015

Mulfinger 2018

Rüsch 2014

Sheehan (submitted)

Subtotal $(95 \% \mathrm{Cl})$

$\begin{array}{rrrrrrr}2.61 & 1.7 & 53 & 2.68 & 1.83 & 54 & 24.8 \% \\ 2.32 & 1.45 & 46 & 2.34 & 1.46 & 67 & 25.1 \% \\ 3.42 & 1.49 & 39 & 4.02 & 1.75 & 45 & 20.2 \% \\ 2.52 & 1.37 & 39 & 2.63 & 1.38 & 47 & 20.8 \% \\ 2.33 & 1.43 & 16 & 3.59 & 1.69 & 19 & 9.0 \% \\ & & 193 & & & 232 & 100.0 \%\end{array}$

df $=4(P=0.28) ; I^{2}=21 \%$

Heterogeneity. Tau $^{2}=0.01 ; \mathrm{Chi}^{2}=5.05, \mathrm{df}=4$
Test for overall effect: $Z=1.56(\mathrm{P}=0.12)$

\section{Depression}

Conley 2020

Corrigan 2015

Mulfinger 2018

Rüsch 2014

Sheehan (submitted)

Subtotal $(95 \% \mathrm{Cl})$

Heterogeneity: Tau $^{2}=0.00 ; \mathrm{Ch}^{2}=1.75, \mathrm{df}=4(\mathrm{P}=0.78) ; \mathrm{I}^{2}=0 \%$

Test for overall effect: $Z=1.09(P=0.27)$
$-0.23[-0.61,0.16]$ $0.14[-0.24,0.52]$ $-0.66[-1.10,-0.21]$ $-0.24[-0.67,0.18]$ $-0.45[-1.12,0.23]$ $-0.26[-0.53,0.02]$

$-0.04[-0.42,0.34]$ $-0.01[-0.39,0.36]$ $-0.36[-0.80,0.07]$ $-0.08[-0.50,0.35]$ $-0.78[-1.47,-0.09]$ $-0.17[-0.39,0.05]$

$-0.07[-0.45,0.31]$ $0.03[-0.34,0.41]$ $-0.11[-0.54,0.32]$ $-0.19[-0.62,0.23]$ $-0.47[-1.15,0.22]$ $-0.11[-0.30,0.08]$
Std. Mean Difference IV, Random, $95 \% \mathrm{Cl}$

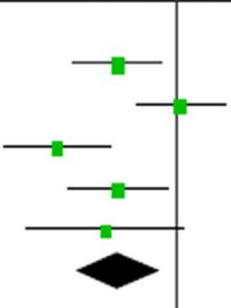


4 Fig. 1 Meta-analysis of HOP effects at the end of the HOP program (post intervention). A negative SMD (to the left) indicates a positive HOP effect, i.e. a reduction of stigma-related variables; except for perceived resources to cope with stigma where an increase (to the right) indicates a positive effect

challenging [50]. In Lausanne, Switzerland, HOP ("Honnête, Ouvert, Prêt", in Engl. "Honest, Open, Ready") was acceptable and feasible for adults with mental illness [51]. One further study from São Paulo, Brazil, among 31 adults with schizophrenia led by Viviane Setti was an uncontrolled pre-post evaluation and therefore not included in our metaanalysis; groups in this study were not peer-led, but run by mental health professionals [52].

\section{Outcomes for meta-analysis}

Two RCTs reported stigma stress (the difference score, see Table 2), but not the underlying subscale scores [21, 47]; two other RCTs only reported the subscale scores, not stigma stress (the difference score [48, 49]). The first RCT had assessed, but not reported, depression after baseline because depressive symptoms were not considered a HOP outcome at that time [47].

To allow a comparison of HOP effects on self-stigma, we chose the only self-stigma measure that was used in all published RCTs, the apply subscale of the Self-Stigma in Mental Illness Scale-Short Form (SSMIS-SF, Table 2); regarding the unpublished 2Share RCT for suicide attempt survivors [46], only the harm subscale of the SSMIS-SF was available which is highly correlated with the apply subscale and has an identical score range [53].

\section{Meta-analysis of HOP efficacy}

For the meta-analysis of HOP efficacy, we looked at three outcomes: (i) stigma stress, including the subscales of perceived stigma-related harm and of perceived coping resources to clarify whether reduced stigma stress was driven by perceptions of increased coping resources and/ or of reduced stigma-related harm; (ii) self-stigma; and (iii) depressive symptoms. For all outcomes, meta-analyses examined the efficacy at the end of the 3-week HOP program (Fig. 1) and, depending on the RCT, at 3- or 4-week followup (Fig. 2). For the two subscales of the Stigma Stress Scale, benefit of the HOP program is indicated by decreased perceived harm and increased coping resources.

Short-term effects at the end of the HOP program showed a positive direction of change, whether or not significant, for all outcomes. Effects were statistically significant for stigma stress with a medium effect size after the intervention (smd $=-0.50,95 \%$-CI -0.87 to -0.14 ; Fig. 1 ), but heterogeneity of effects was high $\left(I^{2}=69 \%\right)$. As evident from the effects on the two subscale scores, reduced stigma stress was mainly driven by significantly increased perceived coping resources to deal with stigma $(\mathrm{smd}=0.55,95 \%$-CI $0.26-0.83$ ) and also by non-significantly reduced perceived stigma-related harm ( $\mathrm{smd}=-0.26,95 \% \mathrm{CI}-0.53$ to 0.02 ; Fig. 1). Effects on self-stigma (smd $=-0.17,95 \%$-CI -0.39 to 0.05 ) and depression $(\mathrm{smd}=-0.11,95 \%-\mathrm{CI}-0.30$ to 0.05 ) at the end of the HOP program were small and statistically not significant, with homogenous effects (Fig. 1).

At follow-up, the meta-analysis revealed a medium effect on stigma stress ( $\mathrm{smd}=-0.40,95 \%$-CI -0.83 to $0.04)$ which did not reach statistical significance and effects were highly heterogeneous $\left(I^{2}=78 \%\right.$; Fig. 2$)$. Here again, the effect was mainly driven by significantly increased coping resources $(\mathrm{smd}=0.58,95 \%$-CI $0.07-1.09)$. The small effect of HOP on self-stigma (smd $=-0.24,95 \%$-CI -0.44 to - 0.04) was statistically significant at follow-up with no heterogeneity (Fig. 2). There was no significant effect on depressive symptoms ( $\mathrm{smd}=-0.09,95 \%$-CI -0.44 to 0.27 ; Fig. 2), with high heterogeneity of effects $\left(I^{2}=67 \%\right)$.

\section{Discussion}

This meta-analysis of five HOP RCTs suggests that HOP is a helpful peer-led intervention to support participants with mental illness in their dealing with stigma. HOP reduced the perception of stigma as a stressor. HOP also had some positive effects on self-stigma, while effects on depressive symptoms were less consistent.

One RCT had reported significantly positive effects on stigma stress appraisals [48]. However, in our meta-analysis, we found no significant effect on subscale appraisals or the stigma stress score for that RCT. This is likely due to the fact that HOP participants in that trial reported substantially more perceived stigma-related harm at baseline than the control group (see Fig. 2 in [48]); after baseline, harm levels of HOP participants approached control group levels. In our meta-analysis, only post-baseline scores were taken into account (see "Methods" above), therefore we did not detect a significant intervention effect on stigma stress appraisals for that trial (Figs. 1 and 2).

This meta-analysis has a number of limitations: the number of RCTs was small and there was no (completed) RCT from a low- or middle-income country; only three outcome domains could be compared across all trials; and there was high heterogeneity between studies. Nevertheless, the metaanalysis provides initial evidence for HOP's efficacy on stigma stress and self-stigma across different target groups and settings.

Future research will need to determine predictors of HOP outcomes. One could speculate, for example, that HOP more effectively reduces depression among women [48] and that 


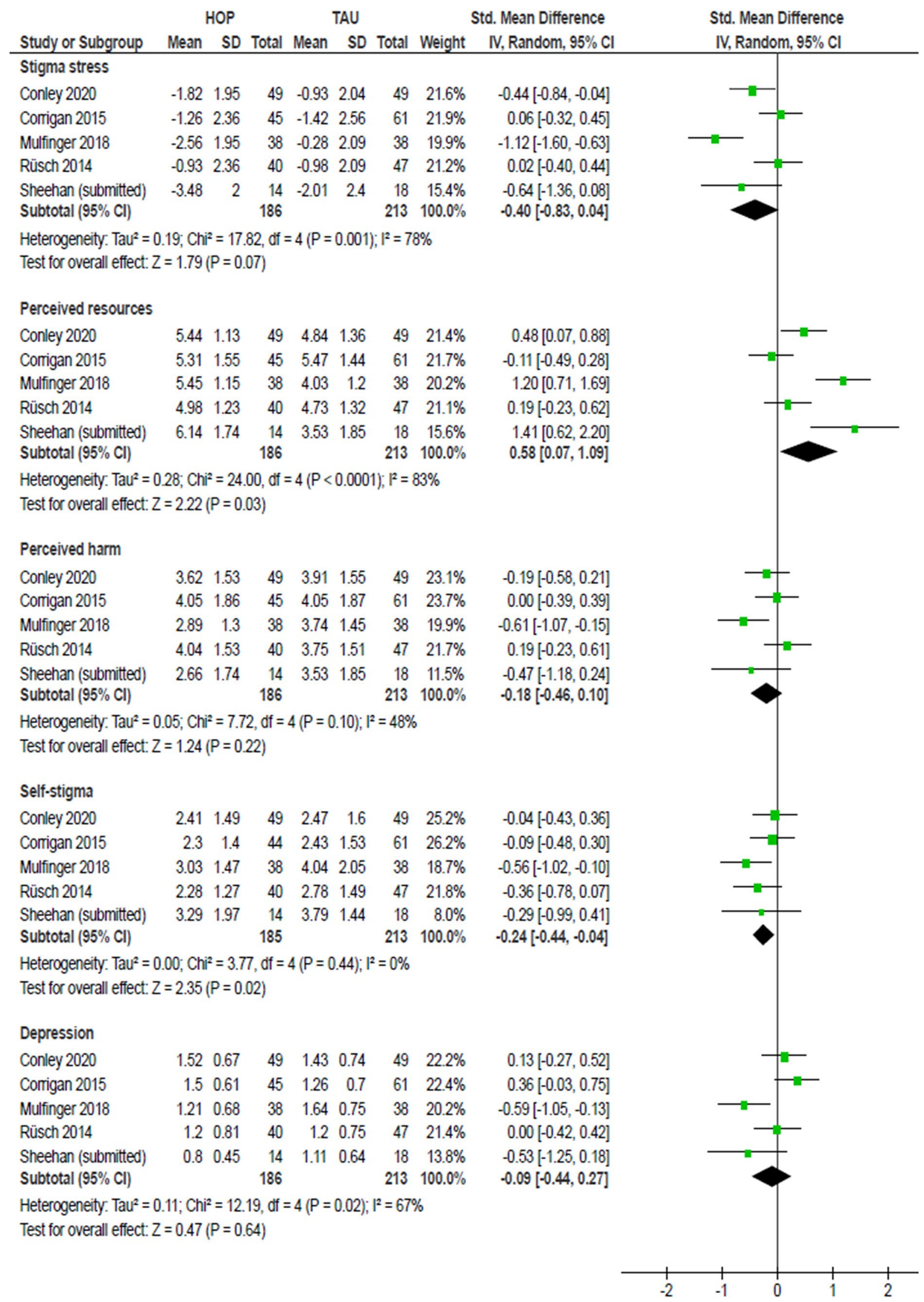


4Fig. 2 Meta-analysis of HOP effects at 3- or 4-week follow-up. A negative SMD (to the left) indicates a positive HOP effect, i.e. a reduction of stigma-related variables; except for perceived resources to cope with stigma where an increase (to the right) indicates a positive effect

HOP may be more effective among young participants whose social networks and disclosure decisions are still more in flux [21]. It should also be examined whether more recent HOP versions that include a fourth booster session [49] have better long-term effects.

\section{Knowledge gaps}

Our findings highlight two major gaps in our knowledge about HOP and its efficacy. First, there are no follow-up data longer than one month after the end of the intervention, and long-term effects are unclear; this information would be important to assess the sustainability of positive effects as well as potential long-term risks for those HOP participants who decide to disclose but may face discrimination later on. Second, the role of actual disclosure decisions is unclear. We do not know whether HOP helps especially those who decide and learn how to disclose strategically; or whether HOP is equally or even more beneficial for participants who choose "empowered non-disclosure".

\section{Implications for outcome measurement}

It can be expected that HOP has proximal effects on stigmarelated variables, such as stigma stress and self-stigma (Table 2). The well-established negative effects of stigma on a person's well-being explain why HOP may also positively affect more distal outcomes, such as quality of life, recovery and depression (see Fig. 2 in [21]). Therefore future trials should measure proximal (stigma-related) outcomes as well as more distal outcomes. Finally, help-seeking requires some degree of disclosure and HOP may improve help-seeking attitudes [21], possibly mediating social and clinical longterm effects of HOP.

At a more technical level, the assessment and reporting of outcomes in future HOP trials could be improved. First, a joint battery of key outcome measures would allow comparisons across trials (see Table 2 for a start). Second, results of the same scale have been reported inconsistently in different trials. Stigma stress is conceptualized as the difference between perceived harm and perceived coping resources, based on Lazarus' transactional model of stress and coping [54]. Stigma stress occurs when people feel that stigmarelated harm exceeds their coping resources. To assess stigma stress, both appraisals (harm and coping resources) need to be taken into account. Rather than both separate appraisals, the key variable to report is the stigma stress difference score, with a range of possible scores from -6 to +6 (Table 2). In two trials, this stress difference score was not reported, only the two underlying subscale scores [48, 49]; in one trial, the perceived harm subscale was erroneously referred to as "stigma as a stressor" [49].

\section{Challenges for HOP evaluation and implementation}

There are a number of challenges to high-quality research on HOP and to broadening the evidence base. First, HOP should be implemented and evaluated as a peer-run program. Peers have gone through disclosure decisions themselves and can be role models for participants. Thus, HOP can be considered a structured, manualized, time-limited mutual support group. It is a misunderstanding to call an intervention "HOP" if groups are run by mental health professionals. This happened in the above-mentioned uncontrolled Brazilian study; due to measurement problems, pre-post changes of stigma stress in this study remained unclear [52].

Second, recruitment to HOP studies and to HOP groups can be a challenge. For example, two German trials of HOP versions had to be ended because recruitment was nearly impossible: One for 2Share in a psychiatric inpatient setting (clinicaltrials.gov/ct2/show/NCT03943862), the other as an online version of Starting the Conversation for parents (clinicaltrials.gov/ct2/show/NCT04107714). In our experience, feedback of nearly all HOP participants is very positive, but many who might be troubled by stigma and disclosure decisions do not attend. This may be related to the intrinsic dilemma that by attending a HOP group you disclose your identity, at least to those in the same group. This led Katrina Scior and colleagues in London, UK, to offer HOP groups for mental health professionals in an anonymous online self-help setting (isrctn.com/ISRCTN18418155; Table 1). Another way to address this problem is to turn HOP into an individual intervention in which one peer worker talks to one participant; Melissa Pyle and colleagues in Manchester, UK, are evaluating their 1-to-1 version of HOP, "Let's Talk", in an ongoing project (Table 1). Results will help to answer the question whether HOP is feasible and effective outside group settings.

Third, to reduce bias due to selective reporting of positive results, future HOP trials should be pre-registered in publicly accessible trial registries with defined primary endpoints as has become the norm for clinical trials.

\section{Future developments}

In the development of new HOP versions for different target groups and cultural contexts, it will be a challenge to keep the balance between flexibility and adaptation on the one hand, and on the other hand, fidelity to the basis of the joint HOP approach. There is an international HOP steering 
committee led by Pat Corrigan that tries to address these issues. In terms of evaluation, there is a lack of long-term follow-up assessments and qualitative research about the experience of HOP participants. It is also unclear whether those participants who decide to disclose have better outcomes than those who decide not to disclose.

By supporting strategic and successful disclosure (i.e. telling the right story to the right people at the right time), HOP has the potential to also reduce public stigma. Disclosure that works well for discloser and recipient means positive contact-arguably the most potent strategy to reduce the public stigma of mental illness $[55,56]$. However, this is a hypothesis that will not be easily tested empirically.

\section{Conclusion}

Based on the available evidence, HOP as a compact peer-led group program appears to be effective and possibly cost-efficient. It can be offered flexibly for different target groups in different settings. HOP supports participants to handle disclosure decisions and thus reduces stigma's impact. Research is needed on its long-term effects. To achieve lasting change in a public health sense, HOP should be combined with programs to reduce public stigma for two reasons: First, people with mental illness must not be left alone in dealing with a stigma for which they are not responsible; second, only decreased public stigma will lead to a less prejudiced social environment that facilitates disclosure, positive contact and social inclusion.

Acknowledgements The authors are grateful to P.W. Corrigan for his comments on a previous version of this manuscript and thank members of the HOP International Steering Committee for their additions to Table 1.

Author contributions Both authors designed the study. NR conducted the conceptual review on HOP, MK the meta-analysis. NR wrote the first draft of the manuscript. Both authors contributed to the manuscript and approved its final version.

Funding Open Access funding enabled and organized by Projekt DEAL. None.

Data availability Not applicable as no primary data was collected.

\section{Declarations}

Conflict of interest NR has translated and adapted the COP/HOP program for German speaking countries.

Open Access This article is licensed under a Creative Commons Attribution 4.0 International License, which permits use, sharing, adaptation, distribution and reproduction in any medium or format, as long as you give appropriate credit to the original author(s) and the source, provide a link to the Creative Commons licence, and indicate if changes were made. The images or other third party material in this article are included in the article's Creative Commons licence, unless indicated otherwise in a credit line to the material. If material is not included in the article's Creative Commons licence and your intended use is not permitted by statutory regulation or exceeds the permitted use, you will need to obtain permission directly from the copyright holder. To view a copy of this licence, visit http://creativecommons.org/licenses/by/4.0/.

\section{References}

1. Link BG, Phelan JC (2001) Conceptualizing stigma. Annu Rev Sociol 27:363-385

2. Mejia-Lancheros C, Lachaud J, Woodhall-Melnik J et al (2020) Longitudinal interrelationships of mental health discrimination and stigma with housing and well-being outcomes in adults with mental illness and recent experience of homelessness. Soc Sci Med 268:113463

3. Rüsch N, Corrigan PW, Waldmann T et al (2018) Attitudes toward disclosing a mental health problem and reemployment: a longitudinal study. J Nerv Ment Dis 206(5):383-385

4. Oexle N, Rüsch N, Viering S et al (2017) Self-stigma and suicidality: a longitudinal study. Eur Arch Psychiatry Clin Neurosci 267(4):359-361

5. Xu Z, Müller M, Lay B et al (2018) Involuntary hospitalization, stigma stress and suicidality: a longitudinal study. Soc Psychiatry Psychiatr Epidemiol 53(3):309-312

6. Rüsch N, Angermeyer MC, Corrigan PW (2005) Mental illness stigma: concepts, consequences, and initiatives to reduce stigma. Eur Psychiatry 20(8):529-539

7. Dubreucq J, Plasse J, Franck N (2021) Self-stigma in serious mental illness: a systematic review of frequency, correlates and consequences. Schizophr Bull. https://doi.org/10.1093/schbul/sbaa181

8. Corrigan PW, Larson JE, Rüsch N (2009) Self-stigma and the "why try" effect: impact on life goals and evidence-based practices. World Psychiatry 8(2):75-81

9. Rüsch N, Müller M, Heekeren K et al (2014) Longitudinal course of self-labeling, stigma stress and well-being among young people at risk of psychosis. Schizophr Res 158(1-3):82-84

10. Rüsch N, Heekeren K, Theodoridou A et al (2015) Stigma as a stressor and transition to schizophrenia after one year among young people at risk of psychosis. Schizophr Res 166(1-3):43-48

11. Rüsch N, Oexle N, Reichhardt L et al (2019) In Würde zu sich stehen-Konzept und Wirksamkeit eines peer-geleiteten Programms zu Offenlegung und Stigmabewältigung [Honest, Open, Proud: Concept and efficacy of a peer-led program to provide support with disclosure decisions and coping with stigma]. Psychiatr Prax 46(2):97-102

12. Corrigan PW, Kosyluk KA, Rüsch N (2013) Reducing self-stigma by coming out proud. Am J Public Health 103(5):794-800

13. Alonso M, Guillén AI, Muñoz M (2019) Interventions to reduce internalized stigma in individuals with mental illness: a systematic review. Span J Psychol 22:e27

14. Tsang HW, Ching SC, Tang KH et al (2016) Therapeutic intervention for internalized stigma of severe mental illness: a systematic review and meta-analysis. Schizophr Res 173(1-2):45-53

15. Lucksted A, Drapalski AL, Brown CH et al (2017) Outcomes of a psychoeducational intervention to reduce internalized stigma among psychosocial rehabilitation clients. Psychiatr Serv 68(4):360-367

16. Drapalski AL, Lucksted A, Brown CH et al (2021) Outcomes of ending self-stigma, a group intervention to reduce internalized stigma, among individuals with serious mental illness. Psychiatr Serv 72(2):136-142 
17. Wood L, Byrne R, Enache G et al (2018) A brief cognitive therapy intervention for internalised stigma in acute inpatients who experience psychosis: a feasibility randomised controlled trial. Psychiatry Res 262:303-310

18. Luoma JB, Kohlenberg BS, Hayes SC et al (2012) Slow and steady wins the race: a randomized clinical trial of acceptance and commitment therapy targeting shame in substance use disorders. J Consult Clin Psychol 80(1):43-53

19. Yanos PT, Lysaker PH, Silverstein SM et al (2019) A randomizedcontrolled trial of treatment for self-stigma among persons diagnosed with schizophrenia-spectrum disorders. Soc Psychiatry Psychiatr Epidemiol 54(11):1363-1378

20. Goffman E (1963) Stigma. Notes on the management of spoiled identity, First Touchstone Edition. A Touchstone Book. Simon \& Schuster, New York

21. Mulfinger N, Müller S, Böge I et al (2018) Honest, Open, Proud for adolescents with mental illness: pilot randomized controlled trial. J Child Psychol Psychiatry 59(6):684-691

22. Xu Z, Lay B, Oexle $\mathrm{N}$ et al (2019) Involuntary psychiatric hospitalisation, stigma stress, and recovery: a 2-year-study. Epidemiol Psychiatr Sci 28(4):458-465

23. Hamann J, Bühner M, Rüsch N (2017) Self-stigma and consumer participation in shared decision making in mental health services. Psychiatr Serv 68(8):783-788

24. Clement S, Schauman O, Graham T et al (2015) What is the impact of mental health-related stigma on help-seeking? A systematic review of quantitative and qualitative studies. Psychol Med 45(1):11-27

25. Perkins A, Ridler J, Browes D et al (2018) Experiencing mental health diagnosis: A systematic review of service user, clinician, and carer perspectives across clinical settings. Lancet Psychiatry 5(9):747-764

26. Lysaker PH, Roe D, Yanos PT (2007) Toward understanding the insight paradox: internalized stigma moderates the association between insight and social functioning, hope, and self-esteem among people with schizophrenia spectrum disorders. Schizophr Bull 33(1):192-199

27. Quinn DM (2018) When stigma is concealable: the costs and benefits for health. In: Major B, Dovidio JF, Link BG (eds) The Oxford handbook of stigma, discrimination, and health. Oxford University Press, Oxford, pp 287-299

28. Rüsch N, Malzer A, Oexle N et al (2019) Disclosure and quality of life among unemployed individuals with mental health problems: a longitudinal study. J Nerv Ment Dis 207(3):137-139

29. Corrigan PW, Lundin R (2001) Don't call me nuts! Coping with the stigma of mental illness. Recovery Press, Chicago

30. Morrow DF (1996) Coming-out issues for adult lesbians: a group intervention. Soc Work 41(6):647-656

31. Pachankis JE (2007) The psychological implications of concealing a stigma: a cognitive-affective-behavioral model. Psychol Bull 133(2):328-345

32. Chaudoir SR, Fisher JD (2010) The disclosure processes model: understanding disclosure decision making and postdisclosure outcomes among people living with a concealable stigmatized identity. Psychol Bull 136(2):236-256

33. Bril-Barniv S, Moran GS, Naaman A et al (2017) A qualitative study examining experiences and dilemmas in concealment and disclosure of people living with serious mental illness. Qual Health Res 27(4):573-583

34. Quinn DM, Williams MK, Quintana F et al (2014) Examining effects of anticipated stigma, centrality, salience, internalization, and outness on psychological distress for people with concealable stigmatized identities. PLoS ONE 9(5):e96977

35. Yanos PT, Lucksted A, Drapalski AL et al (2015) Interventions targeting mental health self-stigma: a review and comparison. Psychiatr Rehabil J 38(2):171-178
36. Wood L, Byrne R, Varese F et al (2016) Psychosocial interventions for internalised stigma in people with a schizophreniaspectrum diagnosis: a systematic narrative synthesis and metaanalysis. Schizophr Res 176(2-3):291-303

37. Xu Z, Huang F, Kösters M et al (2017) Challenging mental health related stigma in China: systematic review and metaanalysis. II. Interventions among people with mental illness. Psychiatry Res 255:457-464

38. Scior K, Rüsch N, White C et al (2020) Supporting mental health disclosure decisions: the Honest, Open, Proud Programme. Br J Psychiatry 216(5):243-245

39. Corrigan PW, Rüsch N, Scior K (2018) Adapting disclosure programs to reduce the stigma of mental illness. Psychiatr Serv 69(7):826-828

40. Deeks JJ, Higgins JPT, Altman DG (2019) Analysing data and undertaking meta-analyses. In: Higgins JPT, Thomas J, Chandler $\mathrm{J}$ et al (eds) Cochrane Handbook for Systematic Reviews of Interventions version 6.1 (updated September 2020). Wiley, Chichester

41. Fu R, Holmer HK (2016) Change score or follow-up score? Choice of mean difference estimates could impact meta-analysis conclusions. J Clin Epidemiol 76:108-117

42. Cochrane Collaboration (2020) Review Manager (RevMan). Version 5.4

43. Mulfinger N, Rüsch N, Bayha P et al (2019) Secrecy versus disclosure of mental illness among adolescents: I. The perspective of adolescents with mental illness. J Ment Health 28(3):296-303

44. Mulfinger N, Rüsch N, Bayha P et al (2019) Secrecy versus disclosure of mental illness among adolescents: II. The perspective of relevant stakeholders. J Ment Health 28(3):304-311

45. Banfield M, Morse AR, Gulliver A (2020) Contextual influences on the impact of a peer worker-led self-stigma program for people with mental health issues: protocol for an interventional implementation science study. Implement Sci Commun 1:26

46. Sheehan L, Oexle N, Bushman M et al. (2020) To Share or Not To Share? Evaluation of a strategic disclosure program for suicide attempt survivors. submitted for publication

47. Rüsch N, Abbruzzese E, Hagedorn E et al (2014) Efficacy of Coming Out Proud to reduce stigma's impact among people with mental illness: pilot randomised controlled trial. Br J Psychiatry 204(5):391-397

48. Corrigan PW, Larson JE, Michaels PJ et al (2015) Diminishing the self-stigma of mental illness by coming out proud. Psychiatry Res 229(1-2):148-154

49. Conley CS, Hundert CG, Charles JLK et al (2020) Honest, Open, Proud-college: effectiveness of a peer-led small-group intervention for reducing the stigma of mental illness. Stigma Health 5(2):168-178

50. Bhatt J, Ruffell TO, Scior K et al (2020) "Who to tell, how and when?" Development and preliminary feasibility of an empowerment intervention for people living with dementia who are fearful of disclosing their diagnosis. Clin Interv Aging 15:1393-1407

51. Pellet J, Suter C, Nguyen A et al (2021) Honnête, ouvert, prêt (HOP)! Acceptabilité et faisabilité de la version française d'un programme d'auto-déstigmatisation de la maladie psychique [Honest, Open, Proud (HOP)! Acceptability and feasibility of the French version of a programme to reduce self-stigma of mental illness]. Ann Med Psychol. https://doi.org/10.1016/j.amp.2020. 08.007

52. Setti VPC, Loch AA, Modelli A et al (2020) Corrigendum to disclosing the diagnosis of schizophrenia: a pilot study of the 'Coming Out Proud' intervention. Int J Soc Psychiatry 66(1):102

53. Corrigan PW, Rafacz J, Rüsch N (2011) Examining a progressive model of self-stigma and its impact on people with serious mental illness. Psychiatry Res 189(3):339-343

54. Lazarus RS, Folkman S (1984) Stress, appraisal, and coping. Springer, New York 
55. Corrigan PW, Morris SB, Michaels PJ et al (2012) Challenging the public stigma of mental illness: a meta-analysis of outcome studies. Psychiatr Serv 63(10):963-973

56. Maunder RD, White FA (2019) Intergroup contact and mental health stigma: a comparative effectiveness meta-analysis. Clin Psychol Rev 72:101749

57. Rüsch N, Corrigan PW, Wassel A et al (2009) A stress-coping model of mental illness stigma: I. Predictors of cognitive stress appraisal. Schizophr Res 110(1-3):59-64

58. Corrigan PW, Michaels PJ, Vega E et al (2012) Self-stigma of mental illness scale-short form: reliability and validity. Psychiatry Res 199:65-69

59. Ritsher JB, Otilingam PG, Grajales M (2003) Internalized stigma of mental illness: psychometric properties of a new measure. Psychiatry Res 121(1):31-49

60. Boyd JE, Otilingam PG, DeForge BR (2014) Brief version of the Internalized Stigma of Mental Illness (ISMI) scale: psychometric properties and relationship to depression, self esteem, recovery orientation, empowerment, and perceived devaluation and discrimination. Psychiatr Rehabil J 37(1):17-23

61. Kohout FJ, Berkman LF, Evans DA et al (1993) Two shorter forms of the CES-D (Center for Epidemiological Studies Depression) depression symptoms index. J Aging Health 5(2):179-193

62. Hautzinger M, Bailer M (1993) Allgemeine Depressionsskala. Beltz, Weinheim

63. Ravens-Sieberer U, Erhart M, Rajmil L et al (2010) Reliability, construct and criterion validity of the KIDSCREEN-10 score: a short measure for children and adolescents' well-being and healthrelated quality of life. Qual Life Res 19(10):1487-1500

64. Wilson CJ, Deane FP, Ciarocchi J et al (2005) Measuring helpseeking intentions: properties of the General Help-Seeking Questionnaire. Can J Couns 39(1):15-28

65. Andresen R, Caputi P, Oades LG (2010) Do clinical outcome measures assess consumer-defined recovery? Psychiatry Res 177(3):309-317 